Pacific

Journal of

Mathematics

COMPARISON RESULTS FOR CONJUGATE AND FOCAL POINTS IN SEMI-RIEMANNIAN GEOMETRY VIA MASLOV INDEX

Miguel Ángel Javaloyes and Paolo Piccione 


\title{
COMPARISON RESULTS FOR CONJUGATE AND FOCAL POINTS IN SEMI-RIEMANNIAN GEOMETRY VIA MASLOV INDEX
}

\author{
Miguel Ángel Javaloyes and PaOlo Piccione
}

\begin{abstract}
We prove an estimate on the difference of Maslov indices relative to the choice of two distinct reference Lagrangians of a continuous path in the Lagrangian Grassmannian of a symplectic space. We discuss some applications to the study of conjugate and focal points along a geodesic in a semiRiemannian manifold.
\end{abstract}

\section{Introduction}

Classical comparison theorems for conjugate and focal points in Riemannian or causal Lorentzian geometry require curvature assumptions, or Morse index arguments; see [Ambrose 1957; Eschenburg and O'Sullivan 1980; Galloway 1979; Kupeli 1986; 1988]. In the general semi-Riemannian world, this approach does not work, because the curvature is never bounded [Dajczer and Nomizu 1980] and the index form has always infinite Morse index. In addition, it is well known that singularities of the semi-Riemannian exponential map may accumulate along a geodesic [Piccione and Tausk 2003], and there is no hope to formulate a meaningful comparison theorem using assumptions on the number of conjugate or focal points.

There are several good indications that a suitable substitute for the notion of size of the set of conjugate or focal points along a semi-Riemannian geodesic is given by the Maslov index. This is a symplectic integer-valued invariant associated to the Jacobi equation, or more generally to the linearized Hamilton equations along the solution of a Hamiltonian system. This number replaces the Morse index of the index form, which in the general semi-Riemannian case is always infinite, and in some nondegenerate cases it is a sort of algebraic count of the conjugate points. In

MSC2000: 53C22, 53C50, 53D12, 53D25.

Keywords: Maslov index, geodesics, semi-Riemannian geometry, conjugate points, focal points.

This work was partially supported by MEC project MTM2007-64504, and Fundación Séneca project 04540/GERM/06, Spain. This research was undertaken within the Programme in Support of Excellence Groups of the Región de Murcia, Spain, by Fundación Séneca, Regional Agency for Science and Technology (Regional Plan for Science and Technology 2007-2010). Javaloyes is partially sponsored by Regional J. Andalucía Grant P06-FQM-01951 and Piccione by Capes (Brazil), Grant BEX 1509-08-0, and Fundación Séneca grant 09708/IV2/08, Spain. 
the Riemannian or causal Lorentzian case, the Maslov index of a geodesic relative to some fixed Lagrangian coincides with the number of conjugate (or focal) points counted with multiplicity. The exponential map is not locally injective around nondegenerate conjugate points [Warner 1965], or more generally around conjugate points whose contribution to the Maslov index is nonzero [Piccione et al. 2004].

Inspired by [Lytchak 2008], in this paper we prove an estimate on the difference between Maslov indices (Proposition 3.3), and we apply this estimate to obtain a number of results that are the semi-Riemannian analogue of the standard comparison theorems in Riemannian geometry (Section 4). These results relate the existence and the multiplicity of conjugate and focal points with the values of Maslov indices naturally associated to a given geodesic. It is very interesting to observe that Riemannian versions of the results proved in the present paper, which are mostly well known, are obtained here with a proof that appears to be significantly more elementary than the classical proof using Morse theory. Detecting conjugate points is interesting because their presence implies multiplicity of geodesics between pairs of points. Estimates on the number of geodesics in terms of conjugate points can be done either via Morse theory or via bifurcation theory. Recent results in this direction are available also in the case of non-Riemannian metrics; see for instance [Abbondandolo et al. 2003; Abbondandolo and Majer 2008] for the Morse theory of geodesics in globally hyperbolic Lorentzian manifolds, [Giannoni et al. 2001] for that theory in stationary Lorentzian manifolds, and [Piccione et al. 2004] for the bifurcation theory of geodesics in arbitrary semi-Riemannian manifolds.

As to the applications in Morse theory or bifurcation theory of the results of the present paper, an important observation is in order. It is now well established that in semi-Riemannian geometry there exist conjugate points that do not contribute to the spectral flow of the second variation of the geodesic action functional. The spectral flow is an integer-valued invariant associated to continuous paths of symmetric Fredholm bilinear forms, and it is a natural substitute for the Morse index in strongly indefinite variational problems. Is is known that jumps of the Maslov index detect bifurcation of geodesics [Piccione et al. 2004]. Similarly, for the Morse theory of geodesics, only conjugate points that contribute to the spectral flow are relevant. The conjugate points whose existence is established in the results of this paper are detected via jumps of the Maslov index, which by the semi-Riemannian Morse index theorem is equal to the spectral flow. Thus, our results can indeed be used in Morse theory and in bifurcation theory.

The paper is organized as follows. In Section 2 we recall a few basic facts about the geometry of the Lagrangian Grassmannian $\Lambda$ of a symplectic space $(V, \omega)$, and about the notion of Maslov index for continuous paths in $\Lambda$. We use a generalized notion of Maslov index, which applies to paths with arbitrary endpoints; for paths with endpoints on the Maslov cycle, there are several conventions regarding the 
contribution of the endpoints. Here we adopt a convention slightly different from that in [Robbin and Salamon 1993]; see (2-3), (2-4) and (2-5).

Section 3 contains the estimate (3-1) on the difference of Maslov indices relative to the choice of two arbitrarily fixed reference Lagrangians $L_{0}$ and $L_{1}$. Using the canonical atlas of charts of the Grassmannian Lagrangian and the transition map (2-1), the proof is reduced to studying the index of perturbations of symmetric bilinear forms; see Lemma 3.1 and Corollary 3.2. Several analogous estimates, (3-2) and (3-3), are obtained using properties (2-4) and (2-6) of Hörmander's index.

Section 4 discusses applications to the study of conjugate and focal points along semi-Riemannian geodesics. In Section 4.1, we describe how to obtain Lagrangian paths out of the flow of the Jacobi equation along a geodesic $\gamma:[a, b] \rightarrow M$ and an initial nondegenerate submanifold $\mathscr{P}$ of a semi-Riemannian manifold $(M, g)$. In Lemma 4.1, we give a characterization of which Lagrangian subspaces of the symplectic space $T_{\gamma(a)} M \oplus T_{\gamma(a)} M$ arise from an initial submanifold construction. Section 4.1 proves the comparison results, which include comparison between conjugate and focal points, as well as between conjugate points relative to distinct initial endpoints. We conclude the paper in Section 5 with a few final remarks concerning the question of nondegeneracy of conjugate and focal points.

\section{Preliminaries}

The Lagrangian Grassmannian. Let us consider a symplectic space $(V, \omega)$, with $\operatorname{dim}(V)=2 n$; we will denote by $\operatorname{Sp}(V, \omega)$ the symplectic group of $(V, \omega)$, which is the closed Lie subgroup of GL $(V)$ consisting of all isomorphisms that preserve $\omega$. A subspace $X \subset V$ is isotropic if the restriction of $\omega$ to $X \times X$ vanishes identically; an $n$-dimensional (that is, maximal) isotropic subspace $L$ of $V$ is called a Lagrangian subspace. We denote by $\Lambda$ the Lagrangian Grassmannian of $(V, \omega)$, which is the collection of all Lagrangian subspaces of $(V, \omega)$, and is a compact differentiable manifold of dimension $\frac{1}{2} n(n+1)$. A real-analytic atlas of charts on $\Lambda$ is given as follows. Start with a Lagrangian decomposition $\left(L_{0}, L_{1}\right)$ of $V$, that is, one in which $L_{0}, L_{1} \in \Lambda$ are transverse Lagrangians with $V=L_{0} \oplus L_{1}$. Then denote by $\Lambda^{0}\left(L_{1}\right)$ the open and dense subset of $\Lambda$ consisting of all Lagrangians $L$ transverse to $L_{1}$. A diffeomorphism $\varphi_{L_{0}, L_{1}}$ from $\Lambda^{0}\left(L_{1}\right)$ to the vector space $\mathrm{B}_{\text {sym }}\left(L_{0}\right)$ of all symmetric bilinear forms on $L_{0}$ is defined by $\varphi_{L_{0}, L_{1}}(L)=\left.\omega(T \cdot, \cdot)\right|_{L_{0} \times L_{0}}$, where $T: L_{0} \rightarrow L_{1}$ is the unique linear map whose graph in $L_{0} \oplus L_{1}=V$ is $L$. The kernel of $\varphi_{L_{0}, L_{1}}(L)$ is the space $L \cap L_{0}$.

We will need to express the transition map $\varphi_{L_{1}, L} \circ \varphi_{L_{0}, L}^{-1}$, where $L_{0}, L_{1}, L \in \Lambda$ are three Lagrangians such that $L \cap L_{0}=L \cap L_{1}=\{0\}$. Note that the two charts $\varphi_{L_{0}, L}$ and $\varphi_{L_{1}, L}$ have the same domain. If $\eta: L_{1} \rightarrow L_{0}$ denotes the isomorphism defined as the restriction to $L_{1}$ of the projection $L \oplus L_{0} \rightarrow L_{0}$, then for all $B \in \mathrm{B}_{\text {sym }}\left(L_{0}\right)$ 
we have (see for instance [Piccione and Tausk 2008, Lemma 2.5.4])

$$
\varphi_{L_{1}, L} \circ \varphi_{L_{0}, L}^{-1}(B)=\eta^{*} B+\varphi_{L_{1}, L}\left(L_{0}\right),
$$

where $\eta^{*}$ is the pull-back by $\eta$.

If $\left(L_{0}, L_{1}\right)$ is a Lagrangian decomposition of $V$, there is a bijection between $\Lambda$ and the set of pairs $(P, S)$, where $P \subset L_{1}$ is a subspace and $S: P \times P \rightarrow \mathbb{R}$ is a symmetric bilinear form on $P$; see [Piccione and Tausk 2008, Exercise 1.17]. More precisely, to each pair $(P, S)$ one associates the Lagrangian subspace $L_{P, S}$ defined by

$$
L_{P, S}=\left\{v+w: v \in P, w \in L_{0},\left.\omega(w, \cdot)\right|_{P}+S(v, \cdot)=0\right\} .
$$

Maslov index. Let us recall a few notions related to symmetric bilinear forms; for further details we recommend [Piccione and Tausk 2008]. Given a symmetric bilinear form $B$ on a (finite-dimensional) real vector space $W$, the index of $B$ is defined to be the dimension of a maximal subspace of $W$ on which $B$ is negative definite. The coindex of $B$ is the index of $-B$, and the signature $\operatorname{sign}(B)$ of $B$ is defined to be the difference coindex minus index.

We will now recall briefly the notion of Maslov index for a continuous path $\ell:[a, b] \rightarrow \Lambda$. For a fixed Lagrangian $L_{0} \in \Lambda$, the $L_{0}$-Maslov index $\mu_{L_{0}}(\ell)$ of $\ell$ is the unique integer such that

(a) $\mu_{L_{0}}$ is fixed-endpoint homotopy invariant;

(b) $\mu_{L_{0}}$ is additive by concatenation;

(c) if $\ell([a, b]) \subset \Lambda^{0}\left(L_{1}\right)$ for some Lagrangian $L_{1}$ transverse to $L_{0}$, then

$$
\mu_{L_{0}}(\ell)=n_{+}\left[\varphi_{L_{0}, L_{1}}(\ell(b))\right]-n_{+}\left[\varphi_{L_{0}, L_{1}}(\ell(a))\right] .
$$

See [Giambò et al. 2004] for a similar discussion. Let us denote by $\mu_{L_{0}}^{-}$the $L_{0^{-}}$ Maslov index function relative to the opposite symplectic form $-\omega$ on $V$. The relation between the functions $\mu_{L_{0}}$ and $\mu_{L_{0}}^{-}$is given by

$$
\mu_{L_{0}}^{-}(\ell)=-\mu_{L_{0}}(\ell)+\operatorname{dim}\left(\ell(a) \cap L_{0}\right)-\operatorname{dim}\left(\ell(b) \cap L_{0}\right),
$$

for every continuous path $\ell:[a, b] \rightarrow \Lambda$.

Let us emphasize that, for curves $\ell$ whose endpoints are not transverse to $L_{0}$, there are several conventions for how the endpoints contribute to the Maslov index. For instance, the definition of $L_{0}$-Maslov index $\bar{\mu}_{L_{0}}$ in [Robbin and Salamon 1993] is obtained by replacing (2-3) with

$$
\bar{\mu}_{L_{0}}(\ell)=\frac{1}{2} \operatorname{sign}\left[\varphi_{L_{0}, L_{1}}(\ell(b))\right]-\frac{1}{2} \operatorname{sign}\left[\varphi_{L_{0}, L_{1}}(\ell(a))\right],
$$

in which case the Maslov index takes values in $\frac{1}{2} \mathbb{Z} .{ }^{1}$

\footnotetext{
${ }^{1}$ In this convention, the Maslov index changes sign when one takes the opposite symplectic form.
} 
Given any continuous path $\ell:[a, b] \rightarrow \Lambda$ and any two Lagrangians $L_{0}, L_{0}^{\prime} \in \Lambda$, the difference $\mu_{L_{0}}(\ell)-\mu_{L_{0}^{\prime}}(\ell)$ depends only on $L_{0}$ and $L_{0}^{\prime}$ and the endpoints $\ell(a)$ and $\ell(b)$ of $\ell$. This quantity will be denoted by $\mathfrak{q}\left(L_{0}, L_{0}^{\prime} ; \ell(a), \ell(b)\right)$, and it coincides (up to some factor that is irrelevant here) with the so-called Hörmander index; see [Hörmander 1971, Definition 3.3.2]. The Hörmander index satisfies certain symmetries; we will need that

$$
\mathfrak{q}\left(L_{0}, L_{1} ; L_{0}^{\prime}, L_{1}^{\prime}\right)=-\mathfrak{q}\left(L_{0}^{\prime}, L_{1}^{\prime} ; L_{0}, L_{1}\right) \text { for all } L_{0}, L_{1}, L_{0}^{\prime}, L_{1}^{\prime} \in \Lambda .
$$

The quantity $\tau\left(L_{0}, L_{1}, L_{2}\right)=\mathfrak{q}\left(L_{0}, L_{1} ; L_{2}, L_{0}\right)=-\mathfrak{q}\left(L_{0}, L_{1} ; L_{0}, L_{2}\right)$ coincides (up to some factor) with the Kashiwara index [Lion and Vergne 1980]. The Kashiwara index function determines completely the Hörmander index by the identity

$$
\mathfrak{q}\left(L_{0}, L_{1} ; L_{0}^{\prime}, L_{1}^{\prime}\right)=\tau\left(L_{0}, L_{1}, L_{0}^{\prime}\right)-\tau\left(L_{0}, L_{1}, L_{1}^{\prime}\right)
$$

for all $L_{0}, L_{1}, L_{0}^{\prime}, L_{1}^{\prime} \in \Lambda$,

which is easily proved using property (b) of the Maslov index.

\section{An estimate of the difference of Maslov indices}

Lemma 3.1. Let $B$ and $C$ be symmetric bilinear forms on a (finite dimensional) real vector space $V$. Then $-n_{-}(C) \leq n_{+}(B+C)-n_{+}(B) \leq n_{+}(C)$.

Proof. It suffices to prove the inequality $n_{+}(B+C)-n_{+}(B) \leq n_{+}(C)$; if this holds for every $B$ and $C$, replacing $C$ with $-C$ and $B$ with $B+C$ will yield the other inequality $-n_{-}(C) \leq n_{+}(B+C)-n_{+}(B)$. Choose $W \subset V$ a maximal subspace of $V$ on which $B+C$ is positive definite, so that $\operatorname{dim}(W)=n_{+}(B+C)$, and write $W=W_{+} \oplus W_{-}$, where $\left.B\right|_{W_{+} \times W_{+}}$is positive definite and $\left.B\right|_{W_{-} \times W_{-}}$is negative semidefinite. Since $B+C$ is positive definite on $W$, it follows that $\left.C\right|_{W_{-} \times W_{-}}$must be positive definite, so that $n_{+}\left(\left.C\right|_{W \times W}\right) \geq \operatorname{dim}\left(W_{-}\right)$. Then

$$
\begin{aligned}
n_{+}(B+C)=\operatorname{dim}(W) & =\operatorname{dim}\left(W_{-}\right)+\operatorname{dim}\left(W_{+}\right) \\
& \leq n_{+}\left(\left.C\right|_{W \times W}\right)+n_{+}\left(\left.B\right|_{W \times W}\right) \leq n_{+}(B)+n_{+}(C) .
\end{aligned}
$$

Corollary 3.2. Let $C$ be a fixed symmetric bilinear form on $V$. Then for all $B_{1}, B_{2} \in \mathrm{B}_{\text {sym }}(V)$,

$$
\left|n_{+}\left(B_{1}\right)-n_{+}\left(B_{2}\right)-n_{+}\left(B_{1}+C\right)+n_{+}\left(B_{2}+C\right)\right| \leq n_{-}(C)+n_{+}(C) .
$$

Proposition 3.3. For any continuous curve $\ell:[a, b] \rightarrow \Lambda$ and any pair $L_{0}, L_{1} \in \Lambda$ of Lagrangians, we have

$$
\left|\mu_{L_{0}}(\ell)-\mu_{L_{1}}(\ell)\right| \leq n-\operatorname{dim}\left(L_{0} \cap L_{1}\right) .
$$


Proof. Since $\mu_{L_{0}}(\ell)-\mu_{L_{1}}(\ell)$ depends only on the endpoints $\ell(a)$ and $\ell(b)$, we can assume the existence of a Lagrangian $L \in \Lambda^{0}\left(L_{0}\right) \cap \Lambda^{0}\left(L_{1}\right)$ such that $\ell(t) \in \Lambda^{0}(L)$ for all $t \in[a, b]$. Namely, we choose $L \in \Lambda^{0}\left(L_{0}\right) \cap \Lambda^{0}\left(L_{1}\right) \cap \Lambda^{0}(\ell(a)) \cap \Lambda^{0}(\ell(b))$ (these are dense opens subsets of $\Lambda$, hence their intersection is nonempty!), and replace $\ell$ by any continuous curve in $\Lambda^{0}(L)$ from $\ell(a)$ to $\ell(b)$.

Once we are in this situation, then the Maslov indices of $\ell$ are given by

$$
\begin{aligned}
& \mu_{L_{0}}(\ell)=n_{+}\left[\varphi_{L_{0}, L}(\ell(b))\right]-n_{+}\left[\varphi_{L_{0}, L}(\ell(a))\right], \\
& \mu_{L_{1}}(\ell)=n_{+}\left[\varphi_{L_{1}, L}(\ell(b))\right]-n_{+}\left[\varphi_{L_{1}, L}(\ell(a))\right] .
\end{aligned}
$$

Now consider the isomorphism $\eta: L_{1} \rightarrow L_{0}$ obtained as the restriction to $L_{1}$ of the projection $L \oplus L_{0} \rightarrow L_{0}$; using formula (2-1) for the transition function for the charts $\varphi_{L_{0}, L}$ and $\varphi_{L_{1}, L}$, we have for all $\alpha \in \Lambda^{0}(L)$

$$
\varphi_{L_{1}, L}(\alpha)=\eta^{*}\left(\varphi_{L_{0}, L}(\alpha)+\eta_{*} \varphi_{L_{1}, L}\left(L_{0}\right)\right),
$$

and so $n_{+}\left(\varphi_{L_{1}, L}(\alpha)\right)=n_{+}\left(\varphi_{L_{0}, L}(\alpha)+C\right)$, where $C=\eta_{*} \varphi_{L_{1}, L}\left(L_{0}\right)$ does not depend on $\alpha$. Note that

$$
n_{+}(C)+n_{-}(C)=n-\operatorname{dim}(\operatorname{Ker}(C))=n-\operatorname{dim}\left(L_{0} \cap L_{1}\right) .
$$

Inequality (3-1) is obtained easily from Corollary 3.2 by setting $B_{1}=\varphi_{L_{0}, L}(\ell(b))$ and $B_{2}=\varphi_{L_{0}, L}(\ell(a))$.

Using the symmetry property (2-6) of Hörmander index, we also get this estimate:

Corollary 3.4. For any continuous curve $\ell:[a, b] \rightarrow \Lambda$ and any pair $L_{0}, L_{1} \in \Lambda$ of Lagrangians, we have

$$
\left|\mu_{L_{0}}(\ell)-\mu_{L_{1}}(\ell)\right| \leq n-\operatorname{dim}(\ell(a) \cap \ell(b)) .
$$

Moreover, changing the sign of the symplectic form and using (2-4), one obtains easily the inequalities

$$
\begin{aligned}
& \mid \mu_{L_{0}}(\ell)-\mu_{L_{1}}(\ell)-\operatorname{dim}\left(\ell(a) \cap L_{0}\right)+\operatorname{dim}\left(\ell(a) \cap L_{1}\right) \\
& \quad+\operatorname{dim}\left(\ell(b) \cap L_{0}\right)-\operatorname{dim}\left(\ell(b) \cap L_{1}\right) \mid \leq n-\operatorname{dim}\left(L_{0} \cap L_{1}\right), \\
& \mid \mu_{L_{0}}(\ell)-\mu_{L_{1}}(\ell)-\operatorname{dim}\left(\ell(a) \cap L_{0}\right)+\operatorname{dim}\left(\ell(a) \cap L_{1}\right) \\
& \quad+\operatorname{dim}\left(\ell(b) \cap L_{0}\right)-\operatorname{dim}\left(\ell(b) \cap L_{1}\right) \mid \leq n-\operatorname{dim}(\ell(a) \cap \ell(b)) .
\end{aligned}
$$

\section{Comparison results for conjugate and focal points}

4.1. Geodesics and Lagrangian paths. Let us now look specifically at curves of Lagrangians arising from the Jacobi equation along a semi-Riemannian geodesic. Let $(M, g)$ be a semi-Riemannian manifold of dimension $n$, and let $\nabla$ be the covariant derivative of the Levi-Civita connection of $g$, with curvature tensor chosen 
with the sign convention $R(X, Y)=\left[\nabla_{X}, \nabla_{Y}\right]-\nabla_{[X, Y]}$. We will assume throughout the section that $\gamma:[a, b] \rightarrow M$ is a given geodesic in $M$; when needed, we will also consider extensions of $\gamma$ to a larger interval $\left[a^{\prime}, b^{\prime}\right] \supset[a, b]$. The Jacobi equation along $\gamma$ is given by $(\mathrm{D} / \mathrm{d} t)^{2} V-R(\dot{\gamma}, V) \dot{\gamma}=0$. Consider the flow of the Jacobi equation, which is the family of isomorphisms

$$
\Phi_{t}: T_{\gamma(a)} M \oplus T_{\gamma(a)} M \rightarrow T_{\gamma(t)} M \oplus T_{\gamma(t)} M \quad \text { for } t \in[a, b]
$$

defined by $\Phi_{t}(v, w)=\left(J_{v, w}(t),(\mathrm{D} / \mathrm{d} t) J_{v, w}(t)\right)$, where $J_{v, w}$ is the unique Jacobi field along $\gamma$ satisfying $J(a)=v$ and $(\mathrm{D} / \mathrm{d} t) J(a)=w$. Consider the symplectic form $\omega$ on the space $V=T_{\gamma(a)} M \oplus T_{\gamma(a)} M$ given by $\omega\left(\left(v_{1}, w_{1}\right),\left(v_{2}, w_{2}\right)\right)=$ $g\left(v_{2}, w_{1}\right)-g\left(v_{1}, w_{2}\right)$. For all $t \in[a, b]$, define $L_{0}^{t}=\{0\} \oplus T_{\gamma(t)} M \subset T_{\gamma(t)} M \oplus T_{\gamma(t)} M$ and set $\ell(t)=\Phi_{t}^{-1}\left(L_{0}^{t}\right)$. An immediate calculation shows that $\ell(t)$ is a Lagrangian subspace of $(V, \omega)$, and we obtain in this way a smooth curve $\ell:[a, b] \rightarrow \Lambda(V, \omega)$. Note that

$$
\ell(a)=L_{0}^{a}=: L_{0} .
$$

Now, consider a smooth connected submanifold $\mathscr{P} \subset M$, with $\gamma(a) \in \mathscr{P}$ and $\dot{\gamma}(a) \in T_{\gamma(a)} \mathscr{P}^{\perp}$. $^{2}$ Let us also assume that $\mathscr{P}$ is nondegenerate at $\gamma(a)$, meaning that the restriction of the metric $g$ to $T_{\gamma(a)} \mathscr{P}$ is nondegenerate. ${ }^{3}$ We will denote by $n_{-}(g, \mathscr{P})$ and $n_{+}(g, \mathscr{P})$ respectively the index and the coindex of the restriction of $g$ to $\mathscr{P}$, so that $n_{-}(g, \mathscr{P})+n_{+}(g, \mathscr{P})=\operatorname{dim}(\mathscr{P})$. Let $S$ be the second fundamental form of $\mathscr{P}$ at $\gamma(a)$ in the normal direction $\dot{\gamma}(a)$, seen as a $g$-symmetric operator $S: T_{\gamma(a)} \mathscr{P} \rightarrow T_{\gamma(a)} \mathscr{P}$. We say that a Jacobi field is $\mathscr{P}$-Jacobi if $V(a) \in T_{\gamma(a)} \mathscr{P}$ and $V^{\prime}(a)+S[V(a)] \in T_{\gamma(a)} \mathscr{P}^{\perp}$. An instant $t_{0} \in(a, b]$ is $\mathscr{P}$-focal if there exists a nonzero $\mathscr{P}$-Jacobi field vanishing at $t_{0}$. The multiplicity of a $\mathscr{P}$-focal instant $t_{0}$ is the dimension of the space of $\mathscr{P}$-Jacobi fields vanishing at $t_{0}$. Consider the subspace $L \mathscr{P} \subset V$ defined by

$$
L_{\mathscr{P}}=\left\{(v, w) \in T_{\gamma(a)} M \oplus T_{\gamma(a)} M: v \in T_{\gamma(a)} \mathscr{P}, w+S(v) \in T_{\gamma(a)} \mathscr{P}^{\perp}\right\},
$$

which is just the construction of Lagrangian subspaces described abstractly in (2-2). If $\pi_{1}: T_{\gamma(a)} M \oplus T_{\gamma(a)} M \rightarrow T_{\gamma(a)} M$ is the projection onto the first summand, then $\pi_{1}(L \mathscr{P})=T_{\gamma(a)} \mathscr{P}$ is orthogonal to $\dot{\gamma}(a)$. Conversely:

Lemma 4.1. Let $L \subset T_{\gamma(a)} M \oplus T_{\gamma(a)} M$ be a Lagrangian subspace, and assume that $P=\pi_{1}(L)$ is orthogonal to $\dot{\gamma}(a)$. Then, there exists a smooth submanifold $\mathscr{P}$ orthogonal to $\dot{\gamma}(a)$ such that $L=L_{\mathscr{\rho}}$.

\footnotetext{
${ }^{2}$ The symbol $\perp$ denotes orthogonality with respect to the semi-Riemannian metric $g$.

${ }^{3}$ The assumption of nondegeneracy for the initial submanifold is not strictly necessary for most of the results of the paper. This assumption is used here for two reasons. First, it guarantees that there are no $\mathscr{P}$-focal points on an initial portion of the geodesic $\gamma$. Second, it allows us to write the second fundamental form as a symmetric linear operator on $T_{\gamma(a)} \mathscr{P}$
} 
Proof. Consider the Lagrangian decomposition $\left(L_{0}, L_{1}\right)$ of $T_{\gamma(a)} M \oplus T_{\gamma(a)} M$ given by $L_{0}=\{0\} \oplus T_{\gamma(a)} M$ and $L_{1}=T_{\gamma(a)} M \oplus\{0\}$. Then there exists a symmetric bilinear form $S: P \times P \rightarrow R$ such that $L=L_{P, S}$ as in (2-2). Let $\mathscr{P}_{0} \subset T_{\gamma(a)} M$ be the submanifold given by the graph of the function $P \ni x \mapsto \frac{1}{2} S(x, x) \dot{\gamma}(a) \in P^{\perp}$. The desired submanifold $\mathscr{P}$ is obtained by taking the exponential of a small open neighborhood of 0 in $\mathscr{P}_{0}$. It is easily seen that the tangent space to $\mathscr{P}_{0}$ at 0 is $P$, and since $\mathrm{d}_{\exp }(0)$ is the identity, $T_{\gamma(a)} \mathscr{P}=P$. Moreover, using the fact that the Christoffel symbols of the chart $\exp _{\gamma(a)}$ vanish at 0 , it is easily seen that the second fundamental form of $\mathscr{P}$ at $\gamma(a)$ in the normal direction $\dot{\gamma}(a)$ is $S$.

Let us also consider the space $L_{0}=\{0\} \oplus T_{\gamma(a)} M$, which corresponds to the Lagrangian associated to the trivial initial submanifold $\mathscr{P}=\{\gamma(a)\}$. Then, an instant $t \in] a, b]$ is $\mathscr{P}$-focal along $\gamma$ if and only if $\ell(t) \cap L_{\mathscr{P}} \neq\{0\}$, and the dimension of this intersection equals the multiplicity of $t$ as a $\mathscr{P}$-focal instant. In particular, $t$ is a conjugate instant, that is, $\gamma(t)$ is conjugate to $\gamma(a)$ along $\gamma$ if $\ell(t) \cap L_{0} \neq\{0\}$. Note that

$$
L_{0} \cap L_{\mathscr{P}}=\{0\} \oplus T_{\gamma(a)} \mathscr{P}^{\perp} .
$$

Thus

$$
\operatorname{dim}\left(L_{0} \cap L_{\mathscr{P}}\right)=\operatorname{codim}(\mathscr{P}) .
$$

For all $t \in] a, b]$, consider the space

$$
A_{\mathscr{P}}[t]=\{(\mathrm{D} / \mathrm{d} t) J(t): J \text { is a } \mathscr{P} \text {-Jacobi field along } \gamma \text { with } J(t)=0\},
$$

while for $t=a$ we set $A_{\mathscr{P}}[a]=T_{\gamma(a)} \mathscr{P}^{\perp}$. Note that $\operatorname{dim}\left(A_{\mathscr{P}}[t]\right)=\operatorname{dim}\left(\ell(t) \cap L_{\mathscr{P}}\right)$. When the initial submanifold is just a point, we will use the notation

$$
A_{0}[t]=\{(\mathrm{D} / \mathrm{d} t) J(t): J \text { is a Jacobi field along } \gamma
$$

$$
\text { with } J(a)=0 \text { and } J(t)=0\} \text {, }
$$

$$
A_{0}[a]=T_{\gamma(a)} M .
$$

It is well known that focal or conjugate points along a semi-Riemannian geodesic may accumulate [Piccione and Tausk 2003]; however, nondegenerate conjugate or focal points are isolated. A $\mathscr{P}$-focal point $\gamma(t)$ along $\gamma$ is nondegenerate when the restriction of the metric $g$ to the space $A_{\mathscr{P}}[t]$ is nondegenerate. This is always the case when $g$ is positive definite (that is, Riemannian), or if $g$ has index 1 (that is, Lorentzian) and $\gamma$ is either timelike or lightlike. Also, the initial endpoint $\gamma(a)$, which is always $\mathscr{P}$-focal of multiplicity equal to the codimension of $\mathscr{P}$, is always isolated.

For all $t \in[a, b]$, let us denote by $n_{-}(g, \mathscr{P}, t), n_{+}(g, \mathscr{P}, t)$ and $\sigma(g, \mathscr{P}, t)$ respectively the index, the coindex and the signature of the restriction of $g$ to $A_{\mathscr{P}}[t]$. 
If $\gamma(t)$ is a nondegenerate $\mathscr{P}$-focal point along $\gamma$ with $t \in] a, b$, then $t$ is an isolated instant of nontransversality of the Lagrangians $\ell(t)$ and $L \mathscr{p}$. Its contribution to the Maslov index $\mu_{L_{\mathscr{P}}}(\ell)$, that is, $\mu_{L_{\mathscr{P}}}\left(\left.\ell\right|_{[t-\varepsilon, t+\varepsilon]}\right)$ with $\varepsilon>0$ sufficiently small, is given by the integer $\sigma(g, \mathscr{P}, t)$. The contribution of the initial point to the Maslov index $\mu_{L \mathscr{P}}(\ell)$, which as observed is always nondegenerate, is given by $n_{+}(g, \mathscr{P}, a)$ :

$$
\mu_{L \mathscr{P}}\left(\left.\ell\right|_{[a, a+\varepsilon]}\right)=n_{+}(g, \mathscr{P}, a)=n_{+}(g)-n_{+}(g, \mathscr{P}) .
$$

In particular,

$$
\mu_{L_{0}}\left(\left.\ell\right|_{[a, a+\varepsilon]}\right)=n_{+}(g) .
$$

Moreover, if $\gamma(b)$ is a nondegenerate $\mathscr{P}$-focal point along $\gamma$, then its contribution to $\mu_{L_{\mathscr{P}}}(\ell)$ is equal to $-n_{-}(g, \mathscr{P}, b)$. Thus, when $g$ is Riemannian the Maslov index $\mu_{L_{\mathscr{P}}}\left(\left.\ell\right|_{[a+\varepsilon, b]}\right)$ is the number of $\mathscr{P}$-focal points along $\left.\gamma\right|_{[a, b[}$ counted with multiplicity. The same holds when $g$ is Lorentzian (that is, index equal to 1) and $\gamma$ is timelike. More generally, if all $\mathscr{P}$-focal points along $\gamma$ are nondegenerate, the Maslov index $\mu_{L \mathscr{P}}(\ell)$ is given by the finite sum

$$
\mu_{\mathscr{P}}(\ell)=n_{+}(g)-n_{+}(g, \mathscr{P})+\sum_{t \in] a, b[} \sigma(g, \mathscr{P}, t)-n_{-}(g, \mathscr{P}, b) .
$$

All this follows easily from the following elementary result:

Lemma 4.2. Let $B: I \rightarrow \mathrm{B}_{\mathrm{sym}}(V)$ be a $C^{1}$-curve of symmetric bilinear forms on a real vector space $V$. Assume that $t_{0} \in I$ is a degeneracy instant, and denote by $B_{0}$ the restriction to $\operatorname{Ker}\left(B\left(t_{0}\right)\right)$ of the derivative $B^{\prime}\left(t_{0}\right)$. If $B_{0}$ is nondegenerate, then $t_{0}$ is an isolated degeneracy instant, and for $\varepsilon>0$ sufficiently small,

$n_{+}\left(B\left(t_{0}+\varepsilon\right)\right)-n_{+}\left(B\left(t_{0}\right)\right)=n_{+}\left(B_{0}\right), \quad n_{+}\left(B\left(t_{0}\right)\right)-n_{+}\left(B\left(t_{0}-\varepsilon\right)\right)=-n_{-}\left(B_{0}\right)$.

Lemma 4.2 is used to compute the Maslov index $\mu_{L \mathscr{P}}$ as follows. Given a $\mathscr{P}$-focal instant $t_{0} \in[a, b]$ and a Lagrangian $L_{1}$ transversal to both $L_{\mathscr{P}}$ and $\ell\left(t_{0}\right)$, consider the smooth path $t \mapsto \varphi_{L_{\mathscr{P}}, L_{1}}(\ell(t))$ of symmetric bilinear forms on $L \mathscr{P}$. The kernel of $B\left(t_{0}\right)$ is identified with the space $A_{\mathscr{P}}\left[t_{0}\right]$, and the restriction of the derivative $B^{\prime}\left(t_{0}\right)$ to $\operatorname{Ker}\left(B\left(t_{0}\right)\right)$ with the restriction of the metric $g$ to $A_{\mathscr{P}}\left[t_{0}\right]$; see for instance [Mercuri et al. 2002].

Comparison results. Let us now prove some comparison results for conjugate and focal instants.

Proposition 4.3. Given any interval $[\alpha, \beta] \subset[a, b]$,

$$
\left|\mu_{L_{0}}\left(\left.\ell\right|_{[\alpha, \beta]}\right)-\mu_{L_{\mathscr{P}}}\left(\left.\ell\right|_{[\alpha, \beta]}\right)\right| \leq \operatorname{dim}(\mathscr{P}) .
$$

Proof. It follows readily from Proposition 3.3 and (4-3). 
In particular, we have the following result concerning the existence of conjugate or focal instant along an arbitrary portion of a geodesic.

Corollary 4.4. For any interval $[\alpha, \beta] \subset] a, b]$,

- if $\left|\mu_{L_{0}}\left(\left.\ell\right|_{[\alpha, \beta]}\right)\right|>\operatorname{dim}(\mathscr{P})$, then there is at least one $\mathscr{P}$-focal instant in $[\alpha, \beta]$;

- if $\left|\mu_{L \mathscr{P}}\left(\left.\ell\right|_{[\alpha, \beta]}\right)\right|>\operatorname{dim}(\mathscr{P})$, then there is at least one conjugate instant in $[\alpha, \beta]$.

Proof. By Proposition 4.3, if $\left|\mu_{L_{0}}\left(\left.\ell\right|_{[\alpha, \beta]}\right)\right|>\operatorname{dim}(\mathscr{P})$, then $\left|\mu_{L \mathscr{P}}\left(\left.\ell\right|_{[\alpha, \beta]}\right)\right|>0$. Since $a \notin[\alpha, \beta]$, this implies that there is a $\mathscr{P}$-focal instant in $[\alpha, \beta]$. The second statement is totally analogous.

On the other hand, the absence of conjugate (focal) instants gives an upper bound on the number of focal (conjugate) instants.

Proposition 4.5. If $\gamma$ has no conjugate instant, then

$$
\left.\left.\left|\mu_{L_{\mathscr{P}}}\left(\left.\ell\right|_{[\alpha, \beta]}\right)\right| \leq \operatorname{dim}(\mathscr{P}) \quad \text { for every interval }[\alpha, \beta] \subset\right] a, b\right] .
$$

Similarly, if $\gamma$ has no $\mathscr{P}$-focal instant, then $\left|\mu_{L_{0}}\left(\left.\ell\right|_{[\alpha, \beta]}\right)\right| \leq \operatorname{dim}(\mathscr{P})$.

Proof. If $\gamma$ has no conjugate (respectively, $\mathscr{P}$-focal) instant, then the Maslov index $\mu_{L_{0}}\left(\left.\ell\right|_{[\alpha, \beta]}\right)=0$ (respectively, $\mu_{L_{\mathscr{P}}}\left(\left.\ell\right|_{[\alpha, \beta]}\right)=0$ ) for all $\left.\left.[\alpha, \beta] \subset\right] a, b\right]$.

All the statements above have a much more appealing version in the Riemannian or timelike Lorentzian case, where the "Maslov index" can be replaced by the number of conjugate or focal instants. In this situation, focal and conjugate instants are always nondegenerate and isolated, and without using Morse theory one can prove nice comparison results:

Corollary 4.6. Assume that either $g$ is Riemannian or that $g$ is Lorentzian and $\gamma$ is timelike (in which case $\mathscr{P}$ is necessarily a spacelike submanifold of $M$ ). Denote by $t_{0}$ and top the instants

$$
\begin{aligned}
t_{0} & =\sup \{t \in] a, b]: \text { there are no conjugate instants in }] a, t]\}, \\
t_{\mathscr{P}} & =\sup \{t \in] a, b]: \text { there are no } \mathscr{P} \text {-focal instants in }] a, t]\} .
\end{aligned}
$$

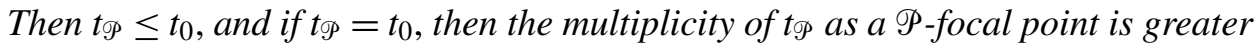
than or equal to its multiplicity as a conjugate point.

Proof. Assume $t_{0}<t_{\circledast} \leq b$ and choose $\left.t^{\prime} \in\right] t_{0}$, $t_{\circledast}[$. Since there are no $\mathscr{P}$-focal instants in $\left.] a, t^{\prime}\right]$ and $\mathscr{P}$ is spacelike, (4-5) implies $\mu_{L_{\mathscr{P}}}\left(\left.\ell\right|_{\left[a, t^{\prime}\right]}\right)=\operatorname{codim}(\mathscr{P})-n_{-}(g)$. On the other hand, $\mu_{L_{0}}\left(\left.\ell\right|_{\left[a, t^{\prime}\right]}\right) \geq n_{+}(g)+1$ since $t_{0}$ is conjugate. Hence,

$$
\mu_{L_{0}}\left(\left.\ell\right|_{\left[a, t^{\prime}\right]}\right)-\mu_{L_{\mathscr{P}}}\left(\left.\ell\right|_{\left[a, t^{\prime}\right]}\right) \geq n_{+}(g)+n_{-}(g)-\operatorname{codim}(\mathscr{P})+1=\operatorname{dim}(\mathscr{P})+1,
$$

which contradicts (4-7). 


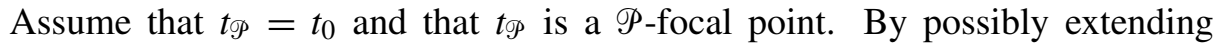
the geodesic $\gamma$ to a slightly larger interval $\left[a, b^{\prime}\right]$ with $b^{\prime}>b$, we can assume the existence of $t^{\prime}>t_{\mathscr{P}}$ with the property that there are no conjugate or $\mathscr{P}_{\text {-focal instants }}$ in $\left.] t_{\mathscr{}}, t^{\prime}\right]$. Then $\mu_{L_{0}}\left(\left.\ell\right|_{\left[a, t^{\prime}\right]}\right)=n_{+}(g)+\operatorname{mul}\left(t_{\mathscr{P}}\right)$, where $\operatorname{mul}\left(t_{\mathscr{P}}\right)$ is the (possibly null) multiplicity of $t_{\mathscr{P}}$ as a conjugate instant. Similarly

$$
\mu_{L_{\mathscr{P}}}\left(\left.\ell\right|_{\left[a, t^{\prime}\right]}\right)=\operatorname{codim}(\mathscr{P})-n_{-}(g)+\operatorname{mul} \phi_{\mathscr{P}}\left(t_{\mathscr{P}}\right),
$$

where mul ${ }_{\mathscr{P}}\left(t_{\mathscr{P}}\right)$ is the multiplicity of $t_{\mathscr{P}}$ as a $\mathscr{P}_{\text {-focal instant. Then }}$

$$
\mu_{L_{0}}\left(\left.\ell\right|_{\left[a, t^{\prime}\right]}\right)-\mu_{L_{\mathscr{P}}}\left(\left.\ell\right|_{\left[a, t^{\prime}\right]}\right)=\operatorname{dim}(\mathscr{P})+\operatorname{mul}\left(t_{\mathscr{P}}\right)-\operatorname{mul}_{\mathscr{P}}\left(t_{\mathscr{P}}\right)
$$

which has to be less than or equal to $\operatorname{dim}(\mathscr{P})$, giving $\operatorname{mul}\left(t_{\mathscr{P}}\right) \geq \operatorname{mul}{ }_{\mathscr{P}}\left(t_{\mathscr{P}}\right)$.

It is known that the result of Corollary 4.6 does not hold without the assumption that the metric $g$ is positive definite or that $g$ is Lorentzian and $\gamma$ timelike. Kupeli [1988, remark in page 585] gives a counterexample by constructing a spacelike geodesic $\gamma$ orthogonal to a timelike submanifold $\mathscr{P}$ of a Lorentzian manifold, with the property that $\gamma$ has conjugate points but no focal point.

In the following statements, $\varepsilon$ will denote a small positive number with the property that there are no conjugate or $\mathscr{P}$-focal instants in $] a, a+\varepsilon]$.

Proposition 4.7. We have $-n_{-}(g, \mathscr{P}) \leq \mu_{L_{\mathscr{P}}}\left(\left.\ell\right|_{[a+\varepsilon, b]}\right)-\mu_{L_{0}}\left(\left.\ell\right|_{[a+\varepsilon, b]}\right) \leq \operatorname{dim} \mathscr{P}$.

Proof. This is a straightforward consequence of formulas (4-5), (4-6) and (4-7) applied on the intervals $[a, b]$ and $[a+\varepsilon, b]$.

In particular, when $g$ is Riemannian, or $g$ is Lorentzian and $\gamma$ timelike, this proposition says that the number of $\mathscr{P}$-focal points along $\gamma$ is greater than or equal to the number of conjugate points along $\gamma$, and that their difference is less than or equal to the dimension of $\mathscr{P}$.

Corollary 4.8. If $\mu_{L_{0}}\left(\left.\ell\right|_{[a+\varepsilon, b]}\right)>n_{-}(g, \mathscr{P})$ or $\mu_{L_{0}}\left(\left.\ell\right|_{[a+\varepsilon, b]}\right)<-\operatorname{dim}(\mathscr{P})$, then

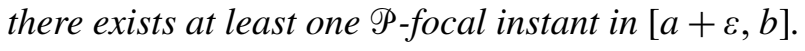

Corollary 4.9. If $\mu_{L_{\mathscr{P}}}\left(\left.\ell\right|_{[a+\varepsilon, b]}\right)<-n_{-}(g, \mathscr{P})$ or $\mu_{L_{\mathscr{P}}}\left(\left.\ell\right|_{[a+\varepsilon, b]}\right)>\operatorname{dim}(\mathscr{P})$, then there exists at least one conjugate instant in $[a+\varepsilon, b]$.

For the following result we need to recall the definition of the space $A_{0}[t]$ given in (4-4); we will denote by $n_{+}(g, t)$ and $n_{-}(g, t)$ respectively the coindex and the index of the restriction of $g$ to $A_{0}[t] \times A_{0}[t]$ and $\operatorname{mul}\left(t_{0}\right)=\operatorname{dim}\left(A_{0}\left[t_{0}\right]\right)$.

The estimate in Corollary 3.4 can be used to obtain results of the following type:

Corollary 4.10. If $\left.\left.t_{0} \in\right] a, b\right]$ is a conjugate instant such that either

$$
\operatorname{mul}\left(t_{0}\right)>n_{-}(g)-\mu_{L_{0}}\left(\left.\ell\right|_{\left[a+\varepsilon, t_{0}\right]}\right) \quad \text { or } \quad \mu_{L_{0}}\left(\left.\ell\right|_{\left[a+\varepsilon, t_{0}\right]}\right)<-n_{+}(g),
$$

then for every $a^{\prime}<a$ there is an instant $t^{\prime} \in\left[a, t_{0}\right]$ such that $\gamma\left(t^{\prime}\right)$ is conjugate to $\gamma(a)$ along $\gamma$. 
Proof. Consider the Lagrangian $L^{\prime} \subset V$ given by

$$
L^{\prime}=\left\{(v, w) \in V: J_{v, w}\left(a^{\prime}\right)=0\right\} .
$$

If there were no instant $t$ in $\left[a, t_{0}\right]$ with $\gamma(t)$ conjugate to $\gamma\left(a^{\prime}\right)$ along $\gamma$, then $\mu_{L^{\prime}}\left(\left.\ell\right|_{\left[a, t_{0}\right]}\right)=\operatorname{dim}\left(L^{\prime} \cap \ell(a)\right)=\operatorname{dim}\left(L^{\prime} \cap \ell\left(t_{0}\right)\right)=0$. By Corollary 3.4, it would then be

$$
\mu_{L_{0}}\left(\left.\ell\right|_{\left[a, t_{0}\right]}\right)=\mu_{L_{0}}\left(\left.\ell\right|_{\left[a, t_{0}\right]}\right)-\mu_{L^{\prime}}\left(\left.\ell\right|_{\left[a, t_{0}\right]}\right) \leq n-\operatorname{mul}\left(t_{0}\right) .
$$

Using (4-6), we get a contradiction with the hypothesis of the corollary. Moreover, using (3-3), we have

$$
\begin{aligned}
\operatorname{mul}\left(t_{0}\right)-n & =\operatorname{dim}\left(\ell(a) \cap \ell\left(t_{0}\right)\right)-n \\
& \leq \mu_{L_{0}}\left(\left.\ell\right|_{\left[a, t_{0}\right]}\right)-\operatorname{dim}\left(\ell(a) \cap L_{0}\right)+\operatorname{dim}\left(\ell(b) \cap L_{0}\right) \\
& =\mu_{L_{0}}\left(\left.\ell\right|_{\left[a, t_{0}\right]}\right)-n+\operatorname{mul}\left(t_{0}\right),
\end{aligned}
$$

that is, $\mu_{L_{0}}\left(\left.\ell\right|_{\left[a, t_{0}\right]}\right) \geq 0$. This together with (4-6) concludes the proof.

When the first conjugate point is nondegenerate, we can state a more precise result.

Corollary 4.11. Let $\left.\left.t_{0} \in\right] a, b\right]$ be the first conjugate instant along $\gamma$, and assume that it is nondegenerate and $\operatorname{mul}\left(t_{0}\right)>n_{-}(g)+n_{-}\left(g, t_{0}\right)$. Then for every $a^{\prime}<a$ there exists and instant $t^{\prime} \in\left[a, t_{0}\right]$ such that $\gamma\left(t^{\prime}\right)$ is conjugate to $\gamma\left(a^{\prime}\right)$ along $\gamma$.

If $g$ is Riemannian, then $n_{-}(g)=n_{-}\left(g, t_{0}\right)=0$, and the result of Corollary 4.11 holds without any assumption of the multiplicity of $t_{0}$.

\section{Final remarks and conjectures}

If the semi-Riemannian manifold $(M, g)$ is real-analytic, then conjugate and focal points do not accumulate along a geodesic, and higher order formulas for the contribution to the Maslov index of each conjugate and focal points are available [Piccione and Tausk 2009]. In this case, the statement of all the above results can be given in terms of the partial signatures of the conjugate and the focal points, which are a sort of generalized multiplicities.

We also observe that the nondegeneracy assumption for the conjugate and focal points is stable by $C^{3}$-small perturbations of the metric, and generic, although a precise genericity statement seems a little involved to prove. We conjecture that, given a differentiable manifold $M$ and a countable set $Z \subset T M$, the set of semiRiemannian metrics $g$ on $M$ having a fixed index and for which all the geodesics $\gamma:[0,1] \rightarrow M$ with $\dot{\gamma}(0) \in Z$ have only conjugate points nondegenerate and of multiplicity equal to 1 is generic. In this situation, the comparison results proved in this paper would have a more explicit statement in terms of number of conjugate and focal points. 
A natural conjecture is also that in the case of stationary Lorentzian metrics, all geodesics have nondegenerate conjugate points whose contribution to the Maslov index is positive and equal to their multiplicity. This fact has been proved in the case of left-invariant Lorentzian metrics on Lie groups of dimension less than 6 [Javaloyes and Piccione 2006] and recently, using semi-Riemannian submersions as in [Caponio et al. 2009], also for spacelike geodesics orthogonal to some timelike Killing vector field. If this conjecture were true in full generality, one would have Riemannian-like comparison results also for spacelike geodesics in stationary Lorentz manifolds.

\section{References}

[Abbondandolo and Majer 2008] A. Abbondandolo and P. Majer, "A Morse complex for Lorentzian geodesics”, Asian J. Math. 12:3 (2008), 299-319. MR 2453558 Zbl 05519473

[Abbondandolo et al. 2003] A. Abbondandolo, V. Benci, D. Fortunato, and A. Masiello, "On the Morse inequalities for geodesics on Lorentzian manifolds”, Math. Res. Lett. 10:4 (2003), 435-445. MR 2004i:58015 Zbl 1056.53042

[Ambrose 1957] W. Ambrose, “A theorem of Myers", Duke Mathematical J. 24 (1957), 345-348. MR 19,680c Zbl 0078.14204

[Caponio et al. 2009] E. Caponio, M. A. Javaloyes, and P. Piccione, "Semi-Riemannian submersions and Maslov index", preprint, 2009. arXiv 0905.0345v1

[Dajczer and Nomizu 1980] M. Dajczer and K. Nomizu, "On the boundedness of Ricci curvature of an indefinite metric", Bol. Soc. Brasil. Mat. 11:1 (1980), 25-30. MR 82d:53039 Zbl 0472.53065

[Eschenburg and O'Sullivan 1980] J.-H. Eschenburg and J. J. O'Sullivan, "Jacobi tensors and Ricci curvature", Math. Ann. 252:1 (1980), 1-26. MR 81k:53037 Zbl 0423.53035

[Galloway 1979] G. J. Galloway, "A generalization of Myers' theorem and an application to relativistic cosmology", J. Differential Geom. 14:1 (1979), 105-116 (1980). MR 81i:53049 Zbl 0444. 53036

[Giambò et al. 2004] R. Giambò, P. Piccione, and A. Portaluri, "On the Maslov index of symplectic paths that are not transversal to the Maslov cycle: Semi-Riemannian index theorems in the degenerate case", preprint, 2004. arXiv math/0306187v3

[Giannoni et al. 2001] F. Giannoni, A. Masiello, P. Piccione, and D. V. Tausk, "A generalized index theorem for Morse-Sturm systems and applications to semi-Riemannian geometry", Asian J. Math. 5:3 (2001), 441-472. MR 2002m:58020 Zbl 1009.58013

[Hörmander 1971] L. Hörmander, "Fourier integral operators, I", Acta Math. 127:1-2 (1971), 79183. MR 52 \#9299 Zbl 0212.46601

[Javaloyes and Piccione 2006] M. A. Javaloyes and P. Piccione, "Conjugate points and Maslov index in locally symmetric semi-Riemannian manifolds", Differential Geom. Appl. 24:5 (2006), 521-541. MR 2007e:53103 Zbl 1111.53039

[Kupeli 1986] D. N. Kupeli, "On existence and comparison of conjugate points in Riemannian and Lorentzian geometry”, Math. Ann. 276:1 (1986), 67-79. MR 87k:53155 Zbl 0585.53037

[Kupeli 1988] D. N. Kupeli, "On conjugate and focal points in semi-Riemannian geometry", Math. Z. 198:4 (1988), 569-589. MR 89g:53099 Zbl 0658.53059

[Lion and Vergne 1980] G. Lion and M. Vergne, The Weil representation, Maslov index and theta series, Progress in Mathematics 6, Birkhäuser, Boston, 1980. MR 81j:58075 Zbl 0444.22005 
[Lytchak 2008] A. Lytchak, "Notes on the Jacobi equation”, preprint, 2008. arXiv 0708.2651v2

[Mercuri et al. 2002] F. Mercuri, P. Piccione, and D. V. Tausk, "Stability of the conjugate index, degenerate conjugate points and the Maslov index in semi-Riemannian geometry", Pacific J. Math. 206:2 (2002), 375-400. MR 2003h:53112 Zbl 1063.53047

[Piccione and Tausk 2003] P. Piccione and D. V. Tausk, "On the distribution of conjugate points along semi-Riemannian geodesics”, Comm. Anal. Geom. 11:1 (2003), 33-48. MR 2005b:53061 Zbl 1077.53059

[Piccione and Tausk 2008] P. Piccione and D. V. Tausk, A student's guide to symplectic spaces, Grassmannians and Maslov index, Instituto de Matemática Pura e Aplicada, Rio de Janeiro, 2008. MR 2463212 Zbl 05530954

[Piccione and Tausk 2009] P. Piccione and D. V. Tausk, "An algebraic theory for generalized Jordan chains and partial signatures in the Lagrangian Grassmannian", Linear and Multilinear Algebra (2009).

[Piccione et al. 2004] P. Piccione, A. Portaluri, and D. V. Tausk, "Spectral flow, Maslov index and bifurcation of semi-Riemannian geodesics", Ann. Global Anal. Geom. 25:2 (2004), 121-149. MR 2005b:53128 Zbl 1050.58015

[Robbin and Salamon 1993] J. Robbin and D. Salamon, "The Maslov index for paths", Topology 32:4 (1993), 827-844. MR 94i:58071 Zbl 0798.58018

[Warner 1965] F. W. Warner, "The conjugate locus of a Riemannian manifold”, Amer. J. Math. 87 (1965), 575-604. MR 34 \#8344 Zbl 0129.36002

Received October 1, 2008. Revised February 13, 2009.

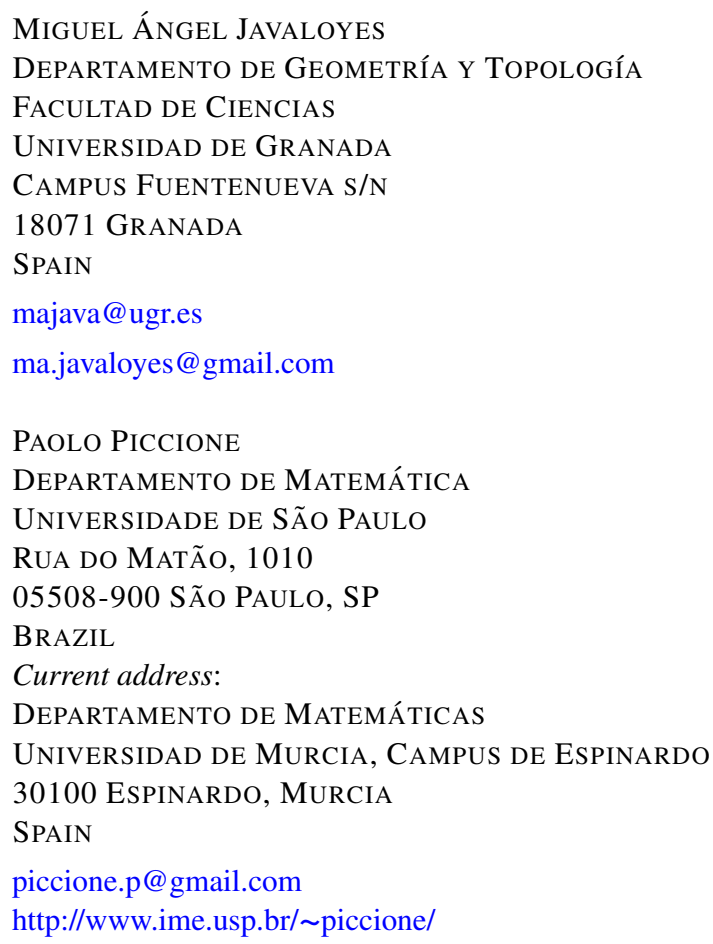

